\title{
Psychological correlates of quality of life in atrial fibrillation
}

Lephuong Ong ${ }^{1}$, Robert Cribbie ${ }^{1}$, Louise Harris ${ }^{2}$, Paul Dorian ${ }^{3}$, David Newman ${ }^{4}$, Iqwal Mangat ${ }^{3}$, Robert Nolan ${ }^{1,2}$ \& Jane Irvine ${ }^{1,2}$

${ }^{1}$ Department of Psychology, York University, 297 BSB, 4700 Keele Street, Toronto, Ontario, M3J 1P3, Canada (E-mail: lephuong@yorku.ca); ${ }^{2}$ University Health Network, Toronto, Canada; ${ }^{3}$ Department of Medicine, St. Michael's Hospital, University of Toronto, Toronto, Canada; ${ }^{4}$ Division of Cardiology, Sunnybrook and Women's College Health Sciences Centre, Toronto, Canada

Accepted in revised form 31 May 2006

\begin{abstract}
Objective: Atrial fibrillation (AF) is associated with significant health-related quality of life (QoL) impairments. Markers of heart disease severity have explained little variance in QoL and it is unclear what other factors will better account for the observed differences in adjustment. We examined whether specific personality traits and illness management styles would help explain the severity of QoL impairments reported in this population. Methods: Patients with $\mathrm{AF}(N=93)$ completed validated questionnaires measuring disease burden (dependent variables: physical and mental QoL, symptom severity, psychological distress), personality (independent variables: anxiety sensitivity, optimism), and illness management style (mediating variable: symptom preoccupation). Hypothesized relationships were evaluated using mediation models. Results: Anxiety sensitivity was associated with poorer physical and mental QoL, greater symptom severity, and higher distress. Optimism was correlated with better mental QoL and lower distress, but unrelated to physical QoL and symptom severity. Symptom preoccupation significantly mediated the relationships between anxiety sensitivity and each of the QoL measures, as well as the relationships between optimism and mental well-being. Conclusions: Personality traits and illness management styles are important to consider when assessing the impact of AF on QoL. The data support a cognitive-behavioral model that explains the direct and indirect relationships between psychological predictors and multiple indices of QoL.
\end{abstract}

Key words: atrial fibrillation, personality, quality of life

Abbreviations: AF - Atrial Fibrillation; QoL - Quality of life; AFSS - Atrial Fibrillation Severity Scale; SF36 - Short Form 36 Health Survey; MCS - Mental Component Score; PCS - Physical Component Score; HADS - Hospital Anxiety and Depression Scale; ASI - Anxiety Sensitivity Index; LOT - Life Orientation Test; IMQ - Illness Management Questionnaire; CI - Confidence Interval

\section{Introduction}

Atrial fibrillation (AF) is a prevalent cardiac arrhythmia that is associated with significant impairments in health-related quality of life (QoL) [1-5]. QoL impairments have been reported to be equal to or greater than that of patients post- angioplasty, post-myocardial infarction, or with congestive heart failure [1]. Clinical variables such as age, beta-blocker use, ventricular dysfunction, and New York Heart Class have accounted for little variance in QoL $[1,6,7]$. QoL impairments persist even in the absence of structural heart disease $[2,8]$. 
Studies have shown that subjective perceptions of illness intrusiveness and the anticipation of experiencing unpleasant symptoms are predictive of adverse outcomes, independent of heart disease severity $[1,2,9]$. Patients' ratings of symptom disruptiveness are also unrelated to the frequency or duration of documented AF symptoms [9]. The apparent lack of association between physical disease severity parameters and QoL in AF patients raises the question of what other factors might explain the severity of QoL impairments observed in this population.

We investigated whether specific psychological factors would help explain the variance in QoL in AF patients. Our theorizing is driven by the cognitive-behavioral framework, which maintains that cognitions, behaviors, and emotions are interdependent $[10,11]$. For instance, maladaptive cognitive appraisals of illness experiences (e.g., "I'm so out of breath that I just can't do anything anymore") may lead to changes in behavior (e.g., reduction in daily activities), which may then affect how patients feel both emotionally and physically (e.g., poorer perceived QoL, greater psychological distress). Poorer perceived QoL and greater psychological distress may in turn perpetuate maladaptive cognitions and behaviors.

In other patient populations, psychological variables have been shown to influence cognitive appraisals of illness experiences and correspond to subjective perceptions of disease burden [12-14]. A similar process may operate in AF patients; psychological characteristics give rise to differential cognitive processing styles, which affect the perceived arrhythmia burden. In this study, disease burden was defined as patients' ratings of their QoL, AF symptom severity, and psychological distress. The psychological characteristics examined in this study were anxiety sensitivity, dispositional optimism, and symptom preoccupation.

Anxiety sensitivity, a fear of anxiety sensations [15], is associated with increased vigilance for physical symptoms, a propensity to over-react to somatic sensations, and increased worry about illness $[16,17]$. Individuals high in anxiety sensitivity interpret anxiety-related sensations as signifying imminent harm and catastrophe, while those low in anxiety sensitivity perceive anxiety symptoms as bothersome, but not disastrous [16]. As anxiety sensitivity predisposes to catastrophic interpretations of physical symptoms, AF patients high in anxiety sensitivity may perceive symptoms as especially disruptive and distressing, resulting in hypervigilance and preoccupation with symptoms, decreased threshold for reporting illness burden, and poor QoL.

Variance in QoL may also be explained by optimism, a generalized expectancy of positive outcomes [18]. Optimists who view preferred outcomes as attainable will continue to invest efforts toward achieving those outcomes [18]. Optimists are confident about coping with obstacles and exhibit increased efforts at problem-solving when faced with stressors [18]. Alternatively, pessimists believe desired outcomes are unattainable and use maladaptive strategies (e.g., behavioral disengagement, catastrophizing) to manage crisis situations, leading to greater distress. Optimists also hold strong attentional biases toward positive stimuli, while the reverse is true for pessimists [19], which may explain the different cognitive and behavioral styles of optimists and pessimists.

Optimism has been linked with several positive health parameters following a serious health crisis including more positive mood, increased life satisfaction, faster rate of recovery, and fewer complications after bypass surgery [12, 13]; lower depression scores in patients one year after hospitalization for an acute myocardial infarction or unstable angina [20]; and better QoL in cancer patients [21].

In AF, different levels of optimism may initiate differential cognitive appraisals and selective attentional biases that affect perceptions of $\mathrm{AF}$, shape illness management styles, and consequently impact QoL. Lower optimism may be associated with negative outcome expectancies and an attentional bias towards arrhythmia symptoms, leading to poorer QoL and increased distress.

We hypothesized that high anxiety sensitivity and low optimism would be associated with poorer QoL, greater symptom severity, and heightened distress through cognitive appraisals that give rise to maladaptive illness management styles, such as increased preoccupation with symptoms. Symptom preoccupation has been found to be correlated with behavioral disengagement, functional impairment, anxiety, and depression in patients with chronic fatigue syndrome [22, 23]; and poorer mental QoL, physical QoL, and greater symptom 
burden in cancer patients [24]. Accordingly, we also sought to determine if symptom preoccupation mediates the relationships between (i) anxiety sensitivity and disease burden, and (ii) optimism and disease burden. The hypothesized model is shown in Figure 1.

\section{Method}

\section{Participants}

This cross-sectional study involved a convenience sample of AF patients from two tertiary-care clinics (St. Michael's Hospital, SMH; Toronto General Hospital, TGH) in Toronto, Canada. The protocol was approved by each institution's research ethics board. Eligible participants were identified via medical chart review of current patients (i.e., seen in the last 12 months) by the investigators. All patients who met inclusion criteria were sent a study introductory letter and were subsequently contacted by telephone regarding study participation. Each participant provided written informed consent and was asked to complete a questionnaire package.

Adult patients with a primary diagnosis of AF, normal ventricular function, and at most mild valvular disease were included. Patients who: (i) could not understand English, (ii) had cognitive impairments, (iii) had a history of severe psychiatric illness, (iv) had received an implantable defibrillator, or (v) had acquired AF due to cardiac surgery or other medical conditions (e.g., hyperthyroidism) were excluded.

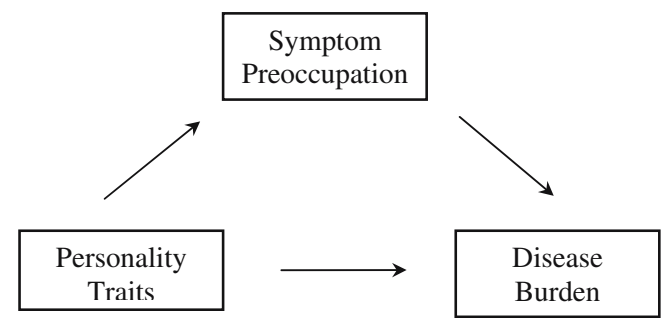

Figure 1. Hypothesized mediation model. Personality traits (anxiety sensitivity, optimism) are hypothesized to be associated with disease burden (mental and physical quality of life, symptom severity, psychological distress). In addition, the relationships between personality traits and disease burden are hypothesized to be mediated by symptom preoccupation.
Information on AF illness duration (i.e., years since AF diagnosis), ventricular function (as indexed by echocardiogram), medications, prior cardioversions, cardiovascular conditions (e.g., valvular disease, hypertension), and non-cardiac medical conditions (e.g., diabetes, cancer) were obtained from patients' medical charts.

\section{Measures}

The Atrial Fibrillation Severity Scale (AFSS) was used to evaluate patient-perceived AF episode frequency (AF burden subscale) and AF symptom severity [25]. The latter is a seven-item checklist that includes common AF symptoms (e.g., dyspnea, dizziness, palpitations). Items are rated on a six-point scale $(0=\mathrm{I}$ have not had this symptom, $5=\mathrm{I}$ have had this symptom a great deal), scores range between 0 and 35, and higher scores indicate greater symptom severity. The symptom severity scale was used as disease-specific indicator of QoL. Published Cronbach's $\alpha$ for AF burden is 0.94 [26]. The symptom severity scale is currently under psychometric evaluation.

Generic QoL was assessed with the Short Form 36 Health Survey (SF-36), which measures health status by evaluating physical, social, and emotional functioning [27]. Items are combined to form the mental (MCS) and physical component summary (PCS). The PCS evaluates energy levels, bodily pain, and interference to social, occupational, or role functioning due to physical health status. The MCS assesses positive affect, distress, and limitations to social, vocational, or role functioning due to emotional difficulties. Scores range from 0 to 100 , where higher scores indicate better functioning. The SF-36 is widely used and normative data is available for healthy and clinical populations. Cronbach's $\alpha$ for the PCS and MCS are 0.93 and 0.88 , respectively, in the general US population [28]. Among AF patients, Cronbach's $\alpha$ is reported to be 0.93 for the PCS and 0.86 for the MCS [29].

The Hospital Anxiety and Depression Scale (HADS) features 14 items developed to detect symptoms of depression and anxiety in medical populations, as it omits the somatic symptoms (e.g., fatigue) that may contribute to falsely elevated scores in medical patients [30]. The depression items relate to loss of interest or pleasure, while the 
anxiety items reflect generalized anxiety. The total score (range: 0-42) was used to measure psychological distress. Items are rated on a four-point scale $(0=$ not at all, $3=$ definitely $) ;$ higher scores indicate greater distress. In AF patients, Cronbach's $\alpha$ are 0.81 and 0.86 for the anxiety and depression subscales, respectively [31]. Cronbach's $\alpha$ for the full scale among cardiac patients is 0.89 [32].

The Anxiety Sensitivity Index (ASI) is a 16-item measure that assesses the fear of anxiety sensations [33] and the extent to which anxiety sensations are believed to cause embarrassment, illness, and loss of control [34]. Items are rated on a five-point scale $(0=$ very little, $4=$ very much $)$ and scores range from 0 to 64 . Higher scores signify greater anxiety sensitivity. Cronbach's $\alpha$ is reported to be 0.88 [33]. This scale has not been validated in AF patients.

The Life Orientation Test (LOT) is a nine-item questionnaire that measures dispositional optimism [14]. Items are rated on a five-point scale $(4=\mathrm{I}$ agree a lot, $0=\mathrm{I}$ disagree a lot), scores range from 0 to 36 , and higher scores denote higher optimism. Cronbach's $\alpha$ is reported to be 0.76 [14]. The LOT has not been validated in AF patients.

The Illness Management Questionnaire (IMQ) assesses four factors: (i) maintaining activity, (ii) illness accommodation, (iii) focusing on symptoms, and (iv) information-seeking [22]. The focusing on symptoms factor, a nine-item scale, was used to measure symptom preoccupation. Items are rated on a six-point scale ( $1=$ never, $6=$ always $)$ and the mean is computed, yielding scores between 1 and 6. Higher scores indicate greater symptom preoccupation. Cronbach's $\alpha$ is 0.88 for the focusing on symptoms factor [22]. Psychometric data for this scale is not available for AF patients.

\section{Analytic strategy}

Baron and Kenny [35] define a mediator as a variable that accounts for the relationship between a variable and an outcome, while a moderator is a variable that alters the relationship between a variable and an outcome. Mediation is more consistent with our hypotheses, as symptom preoccupation is proposed to account for the relationships between the independent and dependent variables of interest. To demonstrate mediation, the following must be established: (i) the independent variable is related to the mediator, (ii) the independent variable affects the dependent variable, (iii) the mediator is associated with the dependent variable, (iv) the relationship between the independent variable and dependent variable is reduced once the mediator is controlled [35].

A series of regression analyses were performed to assess each mediation model. Each model contained one independent variable (either anxiety sensitivity or optimism), one mediator (symptom preoccupation), and one of four dependent variables (physical quality of life, mental quality of life, symptom severity, psychological distress). Control variables were entered at the first step of each model. We assessed all four measures of disease burden as each represents a unique aspect of patient experience, reflecting general physical and mental health status, AF-specific burden, and negative well-being. The analytic strategy is outlined in Table 1.

Univariate relationships were assessed with the Welch $t$-test and Pearson's correlation. The significance of the indirect effect (i.e., the effect of the independent variable on the dependent variable through the mediator) was tested using a

Table 1. Analytic strategy for the mediation analyses

\begin{tabular}{cl}
\hline Variables entered & \\
\hline $\begin{array}{c}\text { Model } 1^{\mathrm{a}} \\
\text { Step 1 }\end{array}$ & $\begin{array}{l}\text { Concurrent medical conditions } \\
\text { AF frequency } \\
\text { Gender }\end{array}$ \\
Step 2 & $\begin{array}{l}\text { Independent variable } \\
\text { c }\end{array}$ \\
Model 2 & Concurrent medical conditions \\
Step 1 & AF frequency \\
Gender & Symptom preoccupation \\
Step 2 & Concurrent medical conditions \\
Full Model & AF frequency \\
Step 1 & Gender \\
& Symptom preoccupation \\
Step 2 & Independent variable
\end{tabular}

Note. ${ }^{\text {a }}$ The dependent variable was one of: physical quality of life, mental quality of life, symptom severity, or psychological distress; ${ }^{\mathrm{b}}$ Cardiovascular or non-cardiovascular; ${ }^{\mathrm{c}}$ Either anxiety sensitivity or optimism; ${ }^{\mathrm{d}} \mathrm{A}$ relationship between the independent variable and the mediator must also be demonstrated; AF, atrial fibrillation. 
bootstrapping procedure to estimate the confidence intervals $(\mathrm{CI})$ of the indirect effect $[36,37]$. This procedure is more robust than the traditional Sobel test recommended by Baron and Kenny [35] with smaller samples as it does not rely on distributional assumptions. In this study, we took 5000 bootstrapped samples (sampling with replacement) to construct the $95 \%$ CI of the true indirect effect.

Based on theoretical considerations and past findings, we controlled for the presence of any concurrent cardiovascular or non-cardiovascular medical conditions, AF frequency, and gender [1, 6, 38-40]. With one independent variable, one mediator, and adjustment for 3 control variables per model, 90 participants are needed to achieve $80 \%$ power to detect a medium effect size [41]. The following distributions were transformed to restore normality: SF-36 MCS and PCS (reflect and square root), HADS total (square root), AFSS Symptom Severity (logarithm), and IMQ-Focusing on Symptoms (logarithm).

\section{Results}

\section{Patient characteristics}

Out of 189 patients who met study entry criteria, questionnaires were mailed to 125 patients who consented to participation. Of these, 99 packages were returned (participation rate: $79 \%$ ). Six respondents were excluded for the following reasons: (1) severe ventricular dysfunction $(n=1)$, (2) patient could not read English and had the questionnaires translated, despite having provided verbal consent $(n=1),(3)$ attrition $(n=3)$, and (4) hyperthyroidism $(n=1)$. Participants who were included did not differ from individuals who were excluded on any demographic or medical characteristic. The total number of participants included is 93 adults ( $n=41$ from SMH, $\mathrm{n}=52$ from TGH).

The mean age of the sample was $61.88 \pm$ 12.04 years. Participants tended to be male, married, and fairly well-educated (Table 2). Most patients had paroxysmal (59\%) and $41 \%$ had persistent AF. Fifty-six percent had AF with concurrent cardiovascular disease and $44 \%$ had lone AF (Table 3). Twenty-one percent had AF episodes more than once per week, $16 \%$ had episodes between once per week and once per month,
Table 2. Demographic characteristics $(N=93)$

\begin{tabular}{llr}
\hline Characteristic & & $(\mathbf{\%})$ \\
\hline Gender & Male & 66 \\
& Female & 34 \\
Marital Status & Married or partnered & 81 \\
& Separated or divorced & 6 \\
& Widowed & 8 \\
Household & Never married & 5 \\
& Living alone & 13 \\
Education & Not living alone & 87 \\
& Secondary school or less & 55 \\
Employment & Post-secondary education & 45 \\
& Employed & 53 \\
& Unemployed or retired & 47 \\
\hline
\end{tabular}

$32 \%$ had episodes less often than once per month. Subjective mean duration of episodes was one hour or less in $25 \%$, lasting several hours in $25 \%$, and lasting one day or more in $14 \%$. At the time of the assessment, $87 \%$ reported some cardiac symptoms, including palpitations $(51 \%)$, fatigue $(41 \%)$, lightheadedness $(46 \%)$, and chest pain $(30 \%)$.

\section{Impact of clinical variables}

The AF pattern (i.e., paroxysmal versus persistent) or presence of underlying heart disease were not related to any dependent measure (all $p>0.10$ ). Thus, these groups were combined for subsequent

Table 3. Medical History $(N=93)$

\begin{tabular}{lc}
\hline Cardiovascular characteristics & \\
AF Illness duration (years) & $7.45 \pm 6.22$ \\
Paroxysmal AF & $55(59 \%)$ \\
Permanent / persistent AF & $38(41 \%)$ \\
Congestive heart failure & $5(5 \%)$ \\
Coronary artery disease & $14(15 \%)$ \\
Hypertension & $43(46 \%)$ \\
Myocardial infarction & $6(6 \%)$ \\
No predisposing factors (lone AF) & $41(44 \%)$ \\
Current Treatments & \\
Anticoagulants & $77(83 \%)$ \\
Warfarin & $60(65 \%)$ \\
Anti-arrhythmics & $41(44 \%)$ \\
Amiodarone & $18(19 \%)$ \\
Sotalol & $8(9 \%)$ \\
Beta Blockers & $51(55 \%)$ \\
$\geq 1$ prior cardioversion & $51(57 \%)$ \\
Selective serotonin reuptake inhibitors & $4(4 \%)$ \\
Benzodiazepines & $4(4 \%)$ \\
\hline
\end{tabular}

Note. AF, atrial fibrillation. 
analyses. Outcome measures (see Table 4 for descriptive statistics) were unrelated to age, anti-arrhythmic medications, $\beta$-blockers, antidepressant therapy, ventricular function, and prior cardioversions (all $p>0.10$ ).

\section{Correlational analyses}

As shown in Table 5 , anxiety sensitivity was related to poorer mental $(r=-0.26, p=0.011)$ and physical $(r=-0.24, p=0.02)$ QoL, greater symptom severity $(r=0.27, p=0.008)$, and heightened psychological distress $(r=0.41, p<0.001)$. Optimism was correlated with higher mental QoL $(r=0.30, p=0.004)$ and lower psychological distress $(r=-0.37, p<0.001)$. Unexpectedly, optimism was unrelated to physical QoL $(r=0.13$, $p=0.21)$ or symptom severity $(r=-0.09, p=0.42)$.

\section{Mediation analyses}

First, we tested the direct effect of the independent variables on symptom preoccupation. Both anxiety sensitivity and optimism were significantly related to symptom preoccupation (anxiety sensi- tivity: $\beta=0.56, p<0.001$; optimism: $\beta=-0.39$, $p<0.001)$.

Second, the direct effects of the independent variables on the dependent variables were tested. Anxiety sensitivity predicted each dependent measure: physical QoL $(\beta=-0.21, p=0.03)$, mental QoL $(\beta=-0.36, \quad p=0.001)$, symptom severity $(\beta=0.23, p=0.025)$, and psychological distress $(\beta=0.43, p<0.001)$. Optimism was associated with mental QoL $(\beta=0.31, p=0.004)$ and psychological distress $(\beta=-0.40, p<0.001)$. Optimism was unrelated to physical QoL $(\beta=0.11, p=0.26)$ and symptom severity $(\beta=-0.15, p=0.17)$.

Third, we assessed the direct effect of the mediator on the dependent variables. Symptom preoccupation predicted each of the dependent measures: physical QoL $(\beta=-0.40, p<0.001)$, mental QoL $(\beta=-0.47, p<0.001)$, symptom severity $(\beta=0.35, p=0.001)$, and psychological distress $(\beta=0.62, p<0.001)$.

Fourth, we tested the indirect effect of each independent variable on the dependent measures, after accounting for the effect of symptom preoccupation and previously identified control variables.

Table 4. Descriptive statistics for study variables

\begin{tabular}{lrrrr}
\hline Measure & $M$ & SD & Range & Cronbach's $\alpha$ \\
\hline Physical QoL (SF-36 PCS) & 45.31 & 10.37 & $19.06-61.12$ & 0.85 \\
Mental QoL (SF-36 MCS) & 52.52 & 7.80 & $29.32-67.01$ & 0.85 \\
Symptom severity (AFSS) & 7.11 & 6.49 & $0.00-31.00$ & 0.85 \\
Psychological distress (HADS) & 8.19 & 5.71 & $0.00-33.00$ & 0.86 \\
Symptom preoccupation (IMQ-FS) & 2.42 & 0.85 & $1.00-5.33$ & 0.84 \\
Anxiety sensitivity (ASI) & 18.95 & 10.86 & $0.00-43.00$ & 0.88 \\
Optimism (LOT) & 25.85 & 6.88 & $7.00-36.00$ & 0.83 \\
\hline
\end{tabular}

Note. $M$, mean; SD, standard deviation; SF-36, Short Form 36 Health Survey; PCS, Physical Component Summary; MCS, Mental Component Summary; AFSS, Atrial Fibrillation Severity Scale; HADS, Hospital Anxiety and Depression Scale; IMQ-FS, Illness Management Questionnaire, Focusing on Symptoms; ASI, Anxiety Sensitivity Index; LOT, Life Orientation Test.

Table 5. Intercorrelations between study variables $(N=93)$

\begin{tabular}{|c|c|c|c|c|c|c|c|}
\hline Variable & 1 & 2 & 3 & 4 & 5 & 6 & 7 \\
\hline 1. Anxiety sensitivity & - & -0.15 & $0.56 * *$ & $-0.24 *$ & $-0.26^{*}$ & $0.27 * *$ & $0.41 * *$ \\
\hline 2. Optimism & & - & $-0.39 * *$ & 0.13 & $0.30^{* *}$ & -0.09 & $-0.37 * *$ \\
\hline 3. Symptom preoccupation & & & - & $-0.44^{* *}$ & $-0.38 * *$ & $0.34 * *$ & $0.59^{* *}$ \\
\hline 4. Physical QoL & & & & - & 0.00 & $-0.49^{* *}$ & $-0.37 * *$ \\
\hline 5. Mental QoL & & & & & - & $-0.21^{*}$ & $-0.64^{* *}$ \\
\hline 6. Symptom severity & & & & & & - & $0.23^{*}$ \\
\hline 7. Psychological distress & & & & & & & - \\
\hline
\end{tabular}

Note. QoL, quality of life; ${ }^{* *} p<0.01$ (two-tailed); ${ }^{*} p<0.05$ (two-tailed). 
With symptom preoccupation entered as the mediator, anxiety sensitivity was no longer related to any of the dependent variables (all $p>0.05$ ), while symptom preoccupation remained associated with each dependent variable (all $p<0.05$ ). None of the $95 \%$ CI's of the indirect effects contained zero, which indicated that the indirect effect between anxiety sensitivity and each dependent measure, through symptom preoccupation, was significant at the 0.05 level. Altogether, the findings demonstrate that symptom preoccupation significantly mediated the relationships between anxiety sensitivity and each dependent variable.

After symptom preoccupation entered the regression, optimism's effect on mental QoL $(\beta=0.14$, $p=0.19)$ and psychological distress $(\beta=-0.17$, $p=0.07$ ) were reduced. The indirect effect between optimism and each mental well-being measure, through symptom preoccupation, was significant (mental QoL, 95\% CI $=.009-0.043$; psychological distress, $95 \% \mathrm{CI}=-0.057$ to -0.018 ). Our results indicate that symptom preoccupation significantly mediates the association between (i) optimism and mental QoL, and (ii) optimism and psychological distress. As multiple models were tested, just one model is depicted in Figure 2 (see Table 6 for a summary of each tested model).

\section{Discussion}

\section{AF burden}

The PCS and MCS scores of participants are within one standard deviation of reported values for other AF cohorts [26, 42] and consistent with published means for patients with hypertension or

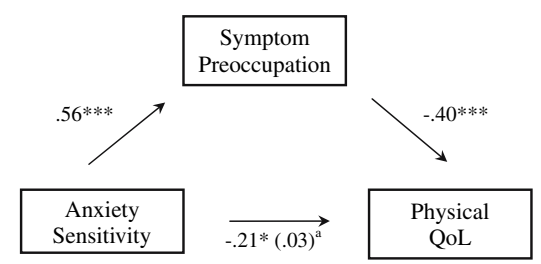

Adj. $R^{2}=.35$

Figure 2. The effect of anxiety sensitivity on physical quality of life after adjustment for the effects of symptom preoccupation. Values represent standardized regression coefficients. ${ }^{\mathrm{a}}$ The effect of anxiety sensitivity on physical quality of life after adjustment for the effects of symptom preoccupation. QoL, quality of life; Adj., adjusted; ${ }^{*} p<0.05$; ${ }^{* *} p<0.001$. recent myocardial infarction [28]. As with previous investigations, factors such as age, ventricular function, $\beta$-blocker use, anti-arrhythmic medications, prior cardioversions, co-existing heart disease, and AF pattern were not significantly associated with QoL or psychological distress [1, 26]. These results reinforce the importance of expanding our focus beyond physical disease parameters to include assessments of personality traits and illness management styles when evaluating disease burden in this population.

\section{Anxiety sensitivity}

As predicted, anxiety sensitivity was correlated with poorer mental and physical QoL, greater symptom severity, and increased distress. Path analyses provided compelling evidence that symptom preoccupation significantly mediated the associations between anxiety sensitivity and each of the outcome measures. The tested models explained $19-40 \%$ of the variance in $\mathrm{QoL}$ and distress.

These results support the hypothesis that anxiety sensitivity is associated with greater attunement towards physical sensations in AF patients, especially cardiac symptoms considered to have adverse consequences, such as an impending arrhythmia episode. Past experiences with AF may foster greater symptom monitoring, which may increase anxiety levels and produce greater vigilance towards arrhythmia symptoms. Evidence for this process stems from studies that have shown that individuals who are high in anxiety sensitivity exhibit increased attunement to internal stimuli that are anxiety-provoking (e.g., cardiac-related symptoms) $[43,44]$ and selective attention towards stimuli with catastrophic physical (e.g., heart attack) consequences $[45,46]$. Taken together, it is plausible that an increased fear of anxiety-related stimuli (i.e., AF symptoms) perpetuates a maladaptive cycle of hypervigilance and preoccupation with symptoms, followed by behavioral disengagement from daily activities, culminating in impaired QoL and heightened distress.

\section{Optimism}

Consistent with hypotheses and previous research [12, 20, 47], optimism was associated with 
Table 6. Summary of mediation analyses

\begin{tabular}{|c|c|c|c|c|c|}
\hline Variable & Direct Effect ${ }^{\mathrm{a}}$ & Adjusted Direct Effect ${ }^{\mathrm{b}}$ & Adjusted $\mathrm{R}^{2}$ & Indirect Effect ${ }^{\mathrm{c}}$ & $95 \% \mathrm{CI}$ of the indirect effect $\mathrm{d}^{\mathrm{d}}$ \\
\hline \multicolumn{6}{|l|}{ Predicting physical QoL: } \\
\hline Anxiety sensitivity & $-0.21^{*}$ & 0.03 & 0.35 & -0.028 & -0.045 to -0.014 \\
\hline \multicolumn{6}{|l|}{ Predicting mental QoL: } \\
\hline Anxiety sensitivity & $-0.36 * *$ & -0.14 & 0.21 & -0.020 & -0.033 to -0.008 \\
\hline Optimism & $0.31^{* *}$ & 0.14 & 0.21 & 0.024 & 0.009 to 0.043 \\
\hline \multicolumn{6}{|c|}{ Predicting symptom Severity: } \\
\hline Anxiety sensitivity & $0.23 *$ & 0.05 & 0.19 & 0.007 & 0.002 to 0.013 \\
\hline \multicolumn{6}{|c|}{ Predicting psychological distress: } \\
\hline Anxiety sensitivity & $0.43 * * *$ & 0.12 & 0.40 & 0.031 & 0.019 to 0.047 \\
\hline Optimism & $-0.40 * * *$ & -0.17 & 0.41 & -0.035 & -0.057 to -0.018 \\
\hline
\end{tabular}

Note. ${ }^{a}$ Effect of the independent variable on the dependent variable; ${ }^{b}$ Effect of the independent variable on the dependent variable, after adjusting for the effects of the mediator, symptom preoccupation; ${ }^{c}$ Indirect effect of the independent variable on the dependent variable via the mediator; ${ }^{\mathrm{d}}$ based on 5000 bootstrap samples of $\mathrm{n}=93$, using transformed data. Values of the direct effects represent standardized regression coefficients. CI, confidence interval; QoL, quality of life; ${ }^{*} p<0.05 ;{ }^{* *} p<0.01 ; * * p<0.001$.

better mental QoL and decreased psychological distress. Unexpectedly, optimism was unrelated to physical QoL or symptom severity, which is contrary to research that has shown optimism to be correlated with better physical functioning, like decreased angina [47], faster rate of recovery and fewer complications from cardiac surgery [13], and lower levels of cancer pain [21]. The inconsistencies with past research might be partially due to methodological differences in assessing physical functioning. For instance, Scheier et al. [13] used physiological, behavioral, and caregiver ratings of physical progress. Similarly, Fitzgerald et al. [47] measured angina symptoms using New York Heart Class. Conversely, our measures of physical functioning reflect self-reported physical health status and subjective symptom burden.

Consistent with our study, a recent investigation of cardiac rehabilitation patients that assessed optimism and physical health status using the LOT and SF-36 PCS, also found optimism to be related to mental but not physical functioning [48]. Notwithstanding methodological differences amongst these studies, the inconsistencies in the pattern of results relating optimism to physical QoL suggests that optimism may have a stronger impact on mental than physical functioning since mental well-being may be more amenable to psychological attitudes (e.g., via acceptance or positive reframing).
Symptom preoccupation significantly mediated the correlations between optimism and mental QoL, in addition to the relationship between optimism and psychological distress. The mediation models accounted for 21 and $41 \%$ of the variance in mental QoL and psychological distress, respectively. These findings indicate that symptom preoccupation may be the mechanism that links optimism with better mental QoL and lower distress. For instance, if symptoms such as chest pain and palpitations are negatively valenced for $\mathrm{AF}$ patients, then it would follow, based on past research [19], that optimistic patients would be less focused on these negative aspects of their arrhythmia relative to pessimistic patients. Pessimists expecting negative AF-related outcomes will become excessively focused on symptoms, and report poor emotional adjustment, through a selfperpetuating circle of symptom preoccupation and behavioral withdrawal from usual activities. Alternatively, optimists with positive outcome expectancies about AF will employ more adaptive strategies for managing their arrhythmia, such as accommodating to the symptoms and maintaining daily activities, which will enhance mental and physical adjustment.

\section{Significance}

Our findings indicate that certain personality traits and illness management styles may undermine 
positive adjustment to $\mathrm{AF}$ and its treatment. If replicated, clinical interventions can be developed to provide support and counseling to patients identified as having dispositional risk factors for poor adjustment. The results of the mediation analyses suggest that cognitive-behavioral interventions targeted at reducing symptom preoccupation may promote better QoL.

\section{Study limitations}

First, the cross-sectional design inhibits the ability to establish causal inferences among study variables. Second, the findings are based on a convenience sample, which limits the generalizability of the results. However, our results are still informative as they illustrate the contribution of psychosocial characteristics to QoL, particularly since so little of patients' morbidity can be explained by disease-related factors. Our models explained 19$41 \%$ of the variance in QoL and psychological distress, which represent medium to large effect sizes by Cohen's [49] conventions. Third, all patients were recruited from specialized, tertiary-care facilities, which raises the possibility that study patients differ from patients seen in general cardiology practice with respect to arrhythmia severity, treatment refractoriness, and distress. Notwithstanding this potential limitation to generalizability, the findings are informative of $\mathrm{AF}$ patients who come to be seen in tertiary-care facilities. Fourth, more male patients participated in the study compared to females, however, this is similar to participation rates in other cohorts $[1,2$, 42]. Fifth, since all of the data was collected via mail-in questionnaires, there is no way to ensure that the questionnaires were filled out by the intended recipient. Sixth, the lack of data on ethnicity limits our ability to assess whether study variables (e.g., perceived symptom severity, QoL) differed as a function of cultural expectations. Lastly, the study participants were mostly married, well-educated, and not living alone. Since these demographic variables may influence the psychological variables investigated in this study, it is important to acknowledge that the results may be restricted to patients with this demographic profile. Study findings should be interpreted in light of these limitations and future work should strive to replicate our findings in a prospective design.

\section{Conclusion}

Our findings suggest that personality traits and illness management styles are important to consider when assessing QoL in AF. The data support a cognitive-behavioral model that explains the direct and indirect relationships between psychological predictors and multiple indices of QoL. The current study is significant in that it attempts to address the gap in the research literature involving QoL in AF, which has been predominantly committed to disease-related indicators of QoL.

\section{Acknowledgments}

We wish to thank the staff and patients at Toronto General Hospital and St. Michael's hospital for their assistance on this project. This work has been supported in part by an Ontario Graduate Scholarship and a Social Sciences and Humanities Research Council of Canada Doctoral Fellowship awarded to the first author.

\section{References}

1. Dorian P, Jung W, Newman D, et al. The impairment of health-related quality of life in patients with intermittent atrial fibrillation: Implications for the assessment of investigational therapy. J Am Coll Cardiol 2000; 36: 1303-1309.

2. van den Berg MP, Hassink RJ, Tuinenburg AE, et al. Quality of life in patients with paroxysmal atrial fibrillation and its predictors: Importance of the autonomic nervous system. Eur Heart J 2001; 22: 247-53.

3. Bubien RS, Knotts-Dolson SM, Plumb VJ, Kay GN. Effect of radiofrequency catheter ablation on health-related quality of life and activities of daily living in patients with recurrent arrhythmias. Circulation 1996; 94: 1585-1591.

4. Kay GN, Bubien RS, Epstein AE, Plumb VJ. Effect of catheter ablation of the atrioventricular junction on quality of life and exercise tolerance in paroxysmal atrial fibrillation. Am J Cardiol 1988; 62: 741-744.

5. Fitzpatrick AP, Kourouyan HD, Siu A, et al. Quality of life and outcomes after radiofrequency His-bundle catheter ablation and permanent pacemaker implantation: Impact of treatment in paroxysmal and established atrial fibrillation. Am Heart J 1996; 131: 499-507.

6. Savelieva I, Paquette M, Dorian P, Luderitz B, Camm AJ. Quality of life in patients with silent atrial fibrillation. Heart 2001; 85: 216-217.

7. Dorian P, Mangat I. Quality of life variables in the selection of rate versus rhythm control in patients with atrial fibrillation: Observations from the Canadian Trial of Atrial Fibrillation. Card Electrophysiol Rev 2003; 7: 276-279. 
8. Lonnerholm S, Blomstrom P, Nilsson L, Oxelbark S, Jideus L, Blomstrom-Lundqvist C. Effects of the maze operation on health-related quality of life in patients with atrial fibrillation. Circulation 2000; 101: 2607-2611.

9. Hamer ME, Blumenthal JA, McCarthy EA, Phillips BG, Pritchett EL. Quality-of-life assessment in patients with paroxysmal atrial fibrillation or paroxysmal supraventricular tachycardia. Am J Cardiol 1994; 74: 826-9.

10. Dobson KS, Dozois DJA. Historical and philosophical bases of the cognitive-behavioral therapies. In: Dobson KS (ed.), Handbook of Cognitive-Behavioral Therapies. New York: The Guilford Press, 2001: 3-39.

11. Beck JS. Cognitive Therapy: Basics and Beyond. New York: The Guildford Press, 1995.

12. King KB, Rowe MA, Kimble LP, Zerwic JJ. Optimism, coping, and long-term recovery from coronary artery surgery in women. Res Nurs Health 1998; 21: 15-26.

13. Scheier MF, Magovern GJ, Abbott RA, et al. Dispositional optimism and recovery from coronary artery bypass surgery: The beneficial effects of physical and psychological well-being. J Pers Soc Psychol 1989; 57: 1024-1040.

14. Scheier MF, Carver CS. Optimism, coping, and health: Assessment and implications of generalized outcomes expectancies. Health Psychol 1985; 4: 219-247.

15. Reiss S, McNally RJ. The expectancy model of fear. In: Reiss S, Bootzin RR (eds.), Theoretical Issues in Behavior Therapy. New York: Academic Press, 1985: 107-121.

16. Keogh E, Dillon C, Georgiou G, Hunt C. Selective attentional biases for physical threat in physical anxiety sensitivity. J Anxiety Disord 2001; 15: 299-315.

17. Cox BJ, Fuentes K, Borger SC, Taylor S. Psychopathological correlates of anxiety sensitivity: Evidence from clinical interviews and self-report measures. J Anxiety Disord 2001; 15: 317-332.

18. Scheier MF, Carver CS. Dispositional optimism and physical well-being: The influence of generalized outcome expectancies on health. J Pers 1987; 55: 169-210.

19. Segerstrom SC. Optimism and attentional bias for negative and positive stimuli. Pers Soc Psychol Bull 2001; 27: 1334 1343.

20. Shnek ZM, Irvine J, Stewart D, Abbey S. Psychological factors and depressive symptoms in ischemic heart disease. Health Psychol 2001; 20: 141-145.

21. Allison PJ, Guichard C, Gilain L. A prospective investigation of dispositional optimism as a predictor of healthrelated quality of life in head and neck cancer patients. Qual Life Res 2000; 9: 951-960.

22. Ray C, Wier W, Stewart D, Miller P, Hyde G. Ways of coping with chronic fatigue syndrome: Development of an illness management questionnaire. Soc Sci Med 1993; 37: 385-391.

23. Ray C, Jefferies S, Weir WR. Coping and other predictors of outcome in chronic fatigue syndrome: A 1-year followup. J Psychosom Res 1997; 43: 405-15.

24. Ransom S, Jacobsen PB, Schmidt JE, Andrykowski MA. Relationship of problem-focused coping strategies to changes in quality of life following treatment for early stage breast cancer. J Pain Symptom Manage 2005; 30: 243-53.
25. Maglio C, Sra J, Paquette M, et al. Measuring quality of life and symptom severity in patients with atrial fibrillation. Pacing Clin Electrophysiol 1998; 21: 839.

26. Dorian P, Paquette M, Newman D, et al. Quality of life improves with treatment in the Canadian Trial of Atrial Fibrillation. Am Heart J 2002; 143: 984-990.

27. Ware JE Jr, Sherbourne CD. The MOS 36-item short-form health survey (SF-36): Conceptual framework and item selection. Med Care 1992; 30: 473-483.

28. Ware JE Jr, Kosinski M. SF-36 Physical and Mental Summary Scales: A Manual for Users of Version 1., 2nd ed., Lincoln, Rhode Island: Quality Metric Incorporated, 2003.

29. Kang Y, Bahler R. Health-related quality of life in patients newly diagnosed with atrial fibrillation. Eur J Cardiovasc Nurs 2004; 3: 71-76.

30. Zigmond AS, Snaith RP. The hospital anxiety and depression scale. Acta Psychiatr Scand 1983; 67: 361-370.

31. Middel B, Bouma J, de Jongste $\mathbf{M}$, et al. Psychometric properties of the Minnesota Living with Heart Failure Questionnaire (MLHF-Q). Clin Rehabil 2001; 15: 489-500.

32. Roberts SB, Bonnici DM, Mackinnon AJ, Worcester MC. Psychometric evaluation of the Hospital Anxiety and Depression Scale (HADS) among female cardiac patients. Br J Health Psychol 2001; 6: 373-383.

33. Reiss S, Peterson RA, Gursky DM, McNally RJ. Anxiety sensitivity, anxiety frequency, and the prediction of fearfulness. Behav Res Ther 1986; 24: 1-8.

34. Maller RG, Reiss S. A behavioral validation of the anxiety sensitivity index. J Anxiety Disord 1987; 1: 265-272.

35. Baron RM, Kenny DA. The moderator-mediator variable distinction in social psychological research: Conceptual, strategic, and statistical considerations. J Pers Soc Psychol 1986; 51: 1173-1182.

36. Hayes AF, Preacher KJ. SPSS and SAS macros for bootstrapping indirect effects in multiple mediator models. 2004. Retrieved November 27, 2005, from http:// www.comm.ohio-state.edu/ahayes/SPSS \% 20programs/ indirect.htm.

37. Preacher KJ, Hayes AF. SPSS and SAS procedures for estimating indirect effects in simple mediation models. Behav Res Methods Instrum Comput 2004; 36: 717-31.

38. Paquette M, Roy D, Talajic M, et al. Role of gender and personality on quality-of-life impairment in intermittent atrial fibrillation. Am J Cardiol 2000; 86: 764-8.

39. Bygrave AJ, Waktare JEP, Camm AJ, et al. Gender differences in 'Quality of life' in atrial fibrillation. J Am Coll Cardiol 1999; 33: 104A.

40. Jenkins LS, Eleanor S, Brodsky M, et al. Quality of life in patients with atrial fibrillation: Baseline data from AFFIRM. Circulation 2000; 102: 512-512.

41. Green SB. How many subjects does it take to do a regression analysis? Multivariate Behav Res 1991; 26: 499510.

42. AFFIRM Investigators. Quality of life in atrial fibrillation: The Atrial Fibrillation Follow-up Investigation of Rhythm Management (AFFIRM) study. Am Heart J 2005; 149: 112-120. 
43. Stewart SH, Buffett-Jerrott SE, Kokaram R. Heartbeat awareness and heart rate reactivity in anxiety sensitivity: A further investigation. J Anxiety Disord 2001; 15: 535-553.

44. Sturges LV, Goetsch VL. Psychophysiological reactivity and heartbeat awareness in anxiety sensitivity. J Anxiety Disord 1996; 10: 283-294.

45. Stewart SH, Conrod PJ, Gignac ML, Pihl RO. Selective processing biases in anxiety-sensitive men and women. Cognition Emotion 1998; 12: 105-133.

46. Asmundson GJG, Norton GR. Anxiety sensitivity in patients with physically unexplained chronic back pain: A preliminary report. Behav Res Ther 1995; 33: 771-777.

47. Fitzgerald TE, Prochaska JO, Pransky GS. Health risk reduction and functional restoration following coronary revascularization: A prospective investigation using dynamic stage typology clustering. Int $\mathbf{J}$ Rehabilit Health 2000; 5: 99-116.

48. Corace KM, Endler NS. Psychosocial predictors of cardiac rehabilitation patient's quality of life. J Appl Biobehav Res 2003; 8: 1-26.

49. Cohen J. Statistical Power Analysis for the Behavioral Sciences., 2nd ed., Hillsdale, NJ: Erlbaum, 1988.

Address for Correspondence: Lephuong Ong, Department of Psychology, York University, 297 BSB, 4700 Keele Street, Toronto, Ontario, M3J 1P3, Canada

Phone: +1-416-590-9348; Fax: + 1-416-736-5814

Email: lephuong@yorku.ca 